PAPER • OPEN ACCESS

Titanium reinforced calcium phosphate improves bone formation and osteointegration in ovine calvaria defects: a comparative 52 weeks study

To cite this article: Sara Gallinetti et al 2021 Biomed. Mater. 16035031

View the article online for updates and enhancements.

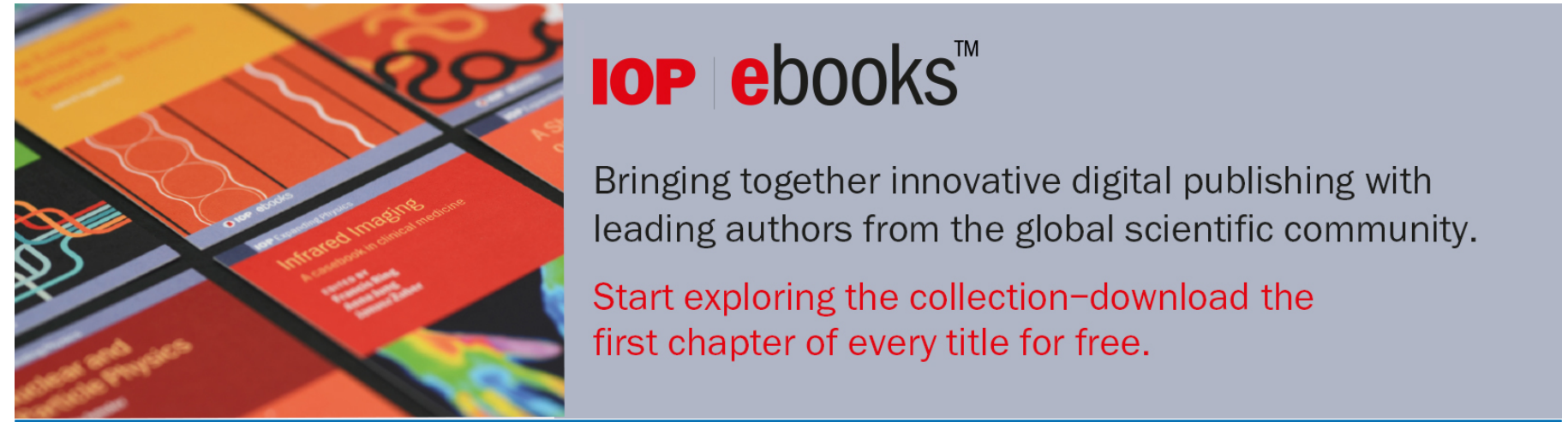

This content was downloaded from IP address 130.238 .188 .37 on $06 / 05 / 2021$ at $16: 52$ 


\title{
Biomedical Materials
}

PAPER

CrossMark

OPEN ACCESS

RECEIVED

24 May 2020

REVISED

30 October 2020

ACCEPTED FOR PUBLICATION

12 November 2020

PUBLISHED

5 March 2021

Original content from

this work may be used

under the terms of the

Creative Commons

Attribution 4.0 licence.

Any further distribution

of this work must

maintain attribution to

the author(s) and the tit

of the work, journal

citation and DOI.

\section{Titanium reinforced calcium phosphate improves bone formation} and osteointegration in ovine calvaria defects: a comparative 52 weeks study

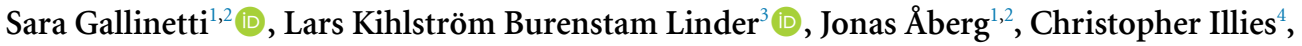 \\ Håkan Engqvist ${ }^{1}$ (D) and Ulrik Birgersson ${ }^{2,5}$ (D) \\ 1 Department of Engineering Sciences, Applied Materials Science Section, Uppsala University, 75121 Uppsala, Sweden \\ 2 OssDsign AB, 75450 Uppsala, Sweden \\ 3 Department of Clinical Neuroscience, Neurosurgical Section, Karolinska University Hospital and Karolinska Institutet, 17176 Stock- \\ holm, Sweden \\ 4 Department of Clinical Pathology, Karolinska University Hospital, Stockholm, Sweden \\ 5 Division of Imaging and Technology, Department of Clinical Science, Intervention and Technology, Karolinska Institutet, 14152 Hud- \\ dinge, Sweden \\ E-mail: ulrik.birgersson@ki.se
}

Keywords: cranial defect, calcium phosphate, titanium mesh, bone formation, immune response

Supplementary material for this article is available online

\section{Abstract}

In a 52 week ovine calvaria implantation model, the restoration of cranial defects with a bare titanium mesh (Ti-mesh) and a titanium mesh embedded in a calcium phosphate (CaP-Ti) were evaluated in seven animals. During the study, no major clinical abnormalities were observed, and all sheep presented a normal neurologic assessment. Blood and cerebrospinal fluid analysis, made at termination, did not show any abnormalities. No indentation of the soft tissue was observed for either test article; however, the Ti-mesh burr-hole covers were associated with filling of the calvarial defect by fibrous tissue mainly. Some bone formation was observed at the bottom of the created defect, but no significant bone was formed in the proximity of the implant. The defect sites implanted with $\mathrm{CaP}-\mathrm{Ti}$ were characterized by a moderate degradation of the calcium phosphate (CaP) that was replaced by mature bone tissue. Calcium-phosphate-filled macrophages were observed in all animals, indicating that they might play a vital role in osteogenesis. The newly formed bone was present, especially at the bony edges of the defect and on the dura side. Integration of the Ti-mesh in a $\mathrm{CaP}$ improved bone formation and osteointegration in comparison to a bare Ti-mesh.

\section{Introduction}

The restoration of cranial defects after craniectomies or craniotomies is a common neurosurgical procedure with defects ranging from small burr-holes up to large cranial defects. Independent of size, the restoration of the cranium should provide adequate protection and restore functionality. Even though there is a wide range of implants available from bone autografts to various alloplastic implants, the optimal reconstructive material still remains undecided [1-3]. The major drawbacks of several alloplastic materials are the slow or lack of osteointegration with the patient's bone and soft tissue related complications, whilst autologous bone flaps generally elicit high rates of resorption over time or cause donor site morbidity [1-4].

Titanium and its alloys remain the most widely used burr-hole covers due to their strength, versatility and generally low production cost $[5,6]$, even though several studies report on compromised soft tissue over time causing wound openings and implant exposure in larger cranial defects [7-10]. One alternative is calcium phosphate $(\mathrm{CaP})$ ceramics that have shown high potential due to their biocompatibility [11], osteoconductivity [12] and ability to develop a direct, adherent and strong bonding with the bone [13]. They are degradable and can be replaced by bone during the healing process. The passive degradation rate is governed by the $\mathrm{CaP}$ phase composition 
and their compositional similarity allows both osteoclasts and macrophages to actively degrade the material. CaPs such as hydroxyapatite have a slow passive degradation rate in vivo [14] which is osteoclastsmediated [15], while brushite and monetite are known to have a faster degradation [16, 17]. Brushite has been widely characterized $[17,18]$ and its active resorption has been connected to macrophage activity [15], while monetite has been less studied. However, monetite appears to be resorbed in vivo faster than the original brushite cements $[19,20]$. Even though CaPs have shown to elicit a promising biological response, their brittle nature has restricted their use to nonload-bearing applications in mechanically stable locations or to applications that require additional osteosynthesis plates and screws [21]. The integration of a titanium skeleton into a $\mathrm{CaP}$ ceramic seems to be a promising way of overcoming the mechanical brittleness of the ceramic material while harnessing its biological performance $[22,23]$. A low clinical complication rate has been observed [22] with an indication that the material is replaced with bone during the healing process for the given $\mathrm{CaP}$ mixture $[22,24,25]$.

These findings warrant a quantitative study to investigate bone formation and remodeling. Therefore, this study aims to compare the biological performance as well as tissue and systemic effects in a 52 weeks ovine calvarial implantation model of a bare titanium mesh (Ti-mesh) against a titanium mesh embedded in a calcium phosphate (CaP-Ti).

\section{Materials and methods}

\subsection{Test articles}

Here, two burr-hole covers were studied and compared in a sheep calvaria model: a bare Ti-mesh burr-hole cover (figures 1 (a) and (c)); and a CaP-Ti (figures 1(b) and (d)).

The Ti-mesh is a laser-cut and anodized medical grade 2 titanium (outer $\varnothing=25 \mathrm{~mm}$, inner $\varnothing=18.5 \mathrm{~mm}$ and $0.4 \mathrm{~mm}$ thick) and produced by Medicon. The burr-hole covers were steam sterilized at $121^{\circ} \mathrm{C}$ for $20 \mathrm{~min}$.

The CaP-Ti burr-hole cover was produced by molding a CaP cement $(\varnothing=13.5 \mathrm{~mm}$ and $4.3 \mathrm{~mm}$ thick) onto a titanium-skeleton with a hexagonal titanium structure (outer $\varnothing=24.6 \mathrm{~mm}$ and $0.4 \mathrm{~mm}$ thick), which allowed the fixation to the sheep calvaria. The ceramic is a combination of Monetite$\beta$-Tricalcium Phosphate-Pyrophosphate[25] and produced starting from monocalcium phosphate (MCPM; Alfa Aesar, Thermo Fisher, Karlsruhe, Germany) and a mixture of beta-tricalcium phosphate $(\beta$-TCP) and dicalcium pyrophosphate powder $(\beta$ CPP, Sigma-Aldrich, Steinheim, Germany) mixed with glycerol which was used to enable molding under controlled conditions. The cement was allowed to harden overnight in sterile water. After removal from the mold, the CaP-Ti burr-hole was left in sterile water for $48 \mathrm{~h}$ to reduce the glycerol content. The burr-hole covers were steam sterilized at $121{ }^{\circ} \mathrm{C}$ for $20 \mathrm{~min}$. The burr-hole covers were fixed on the sheep skulls with self-drilling $1.5 \times 4 \mathrm{~mm}$ bone screws (Titanium Screws for Neuro Fixation System, Medicon).

\subsection{Ceramic characterization}

Samples of the CaP ceramic were prepared, as previously described, for the following characterization methods: hexagonal tiles for porosity, ground powder for $\mathrm{x}$-ray diffraction (XRD) and specific surface area (SSA).

Phase composition was analyzed by XRD (D8 Advance, Bruker AXS GmbH, Karlsruhe, Germany) with a theta-theta $(2 \theta)$ setup and $\mathrm{Ni}$-filtered $\mathrm{Cu}-$ $\mathrm{K}$ irradiation. Diffraction patterns were collected between $2 \theta$ of $20^{\circ}-46^{\circ}$, with steps of $0.02 \mathrm{~s}$ and $0.25 \mathrm{~s}$, with a sample rotation speed of $80 \mathrm{rad} \mathrm{min}^{-1}$ and a beam knife. Rietveld refinements were applied to perform a quantitative phase composition analysis. The BGMN software (www.bgmn.de) was used with Profex as user interface [26]. Crystalline models were taken from Profex database based on literature references: $\beta$-TCP [27], monocalcium phosphate monohydrate (MCPM) [28], brushite [29], $\beta$-calcium pyrophosphate ( $\beta$-CPP) [30] and monetite [31]. No other phases were identified in the diffraction patterns.

The SSA of the ceramic was determined by nitrogen adsorption at $77 \mathrm{~K}$ according to the BrunnauerEmmet-Teller method [32] in an ASAP 2020 (Micromeritics, Norgross, GA). The holder was filled with around $1 \mathrm{~g}$ of ceramic powder. The total porosity of the ceramic was measured on eight hexagonal samples with the Archimedes method [33] in distilled water.

Morphology of CaP was visualized using scanning electron microscopy (SEM) (Marvin, Zeiss, Germany). Prior to scanning the samples were dried in vacuum at $60{ }^{\circ} \mathrm{C}$ for $2 \mathrm{~h}$ and then fixed with carbon tape to the sample holder and coated with Au-Pd. The coating was sputtered for $60 \mathrm{~s}$ at a voltage of $2 \mathrm{kV}$ and current of $20 \mathrm{~mA}$ by mean of Polaron SC7640 Sputter coater (Thermo VG Scientific).

\subsection{In vivo study}

\subsubsection{Animals model}

Seven skeletally mature female sheep (Ovis Aries) of the breed Blanche du Massif Central were used as animal model in the study. Their age ranged between 2.2 years and 3.3 years and their weight varied between $67 \mathrm{~kg}$ and $74 \mathrm{~kg}$. The husbandry conditions of the animals conformed to the European requirements (European Directive 2010/63/EU). The protocol of the study was approved by the NAMSA Ethical Committee (NAMSA, Chasse-sur-Rhône, France). 


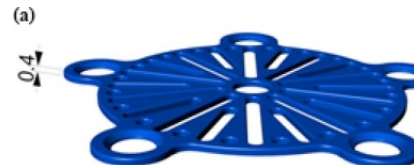

(c)
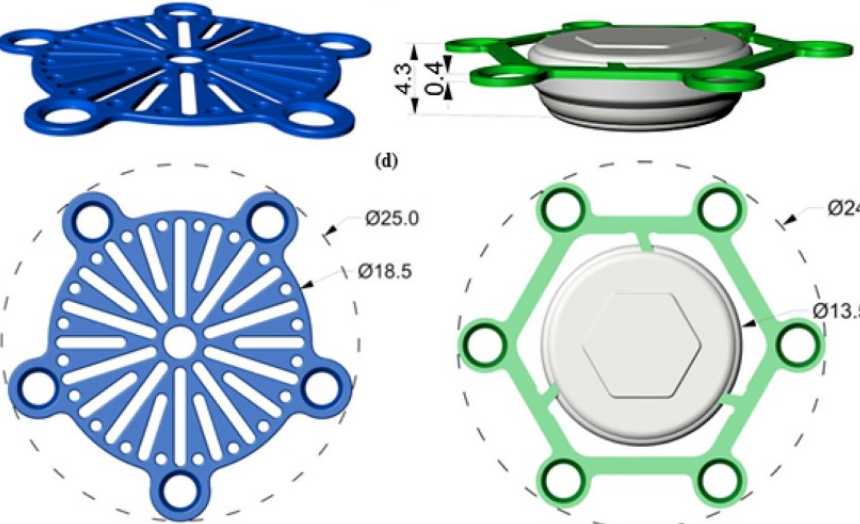

(d)

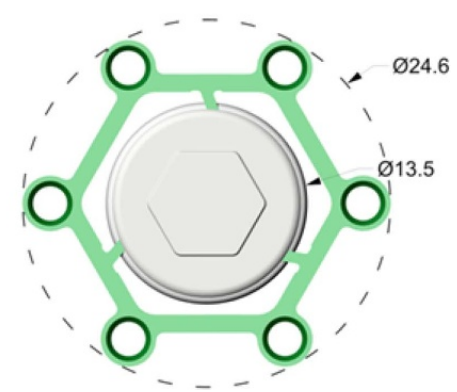

Figure 1. Lateral view of (a) Ti-mesh and (b) CaP-Ti burr-hole covers. Top view of (c) Ti-mesh and (d) CaP-Ti burr-hole covers, including dimensions.

\subsubsection{Surgery}

The surgery was conducted at NAMSA, an accredited GLP facility which is registered with the French Department of Agriculture for animal housing, care and pre-clinical investigations.

Prior to surgery each sheep received an intramuscular injection of a nonsteroidal anti-inflammatory drug flunixine (Meflosyl ${ }^{\circledast}$ Injectable, Zoetis); and as a prophylactic measure antibiotics amoxicillin (Duphamox LA ${ }^{\circledast}$, Zoetis) was administered intramuscularly and enrofloxacin (Baytril ${ }^{\circledR} 10 \%$, Bayer Pharma) subcutaneously. Preanesthetic medication consisted of a mixture of diazepam (Valium ${ }^{\circledR}$, Roche) and butorphanol (Torbugesic ${ }^{\circledR}$, Zoetis) that was administrated intravenously (IV). Anesthesia was induced by IV injection of propofol (Propovet ${ }^{\circledR}$, Axience) and maintained by inhalation of an $\mathrm{O}_{2}-$ Isoflurane mixture (Isoflo ${ }^{\circledast}$, Axience) through a tracheal tube. Following anesthesia, the sheep were placed in ventral recumbency. A midline incision was made through the skin from the right and left orbits to the occipital part of the skull. The temporalis muscles were subperiosteally elevated from the frontal, parietal and occipital bones and retracted bilaterally (flap). All remaining soft tissue attached to the bone was removed from the exposed site.

Two circular defects were created in the frontal bone, bilaterally with a perforator (ZyphrTM Disposable Cranial Perforator, Large 14/11 mm, Stryker) connected to a high-speed drilling unit (Medtronic). Constant irrigation with physiological saline solution was maintained throughout the defect creation to control any temperature increase at the implantation site. The dura mater was left intact and any bone debris was removed. The surgical area was flushed with saline ( $\mathrm{NaCl} 0.9 \%$, Aguettant) to remove bone debris and then dried by aspiration.

In accordance with a predetermined randomization protocol, the left and right frontal burr-hole defects were covered with a CaP-Ti or Ti-Mesh test article and fixated with 3-5 micro screws (Medicon).

The soft tissues were closed with resorbable sutures $\left(\right.$ Vicryl $^{\circledR} 2.0$, Ethicon) and the skin was closed with non-resorbable sutures (Prolene ${ }^{\circledast} 2.0$, Ethicon) and surgical staples (Covidien). The wounds were disinfected with oxytetracycline (Intervet).

Postoperatively, an intramuscular injection of buprenorphine (Buprecare ${ }^{\circledR}$, AXIENCE) was administered once or twice daily for $2 \mathrm{~d}$. Fluxine (Meflosyl ${ }^{\circledR}$ Injectable, Zoetis) was administered intramuscularly daily for $7 \mathrm{~d}$ post-surgery and antibiotics (amoxicillin, Duphamox LA ${ }^{\circledast}$, Zoetis and enrofloxacin, Baytril ${ }^{\circledR}$ $10 \%$, Bayer Pharma) were given for 6 weeks postsurgery, intramuscularly (once every $2 \mathrm{~d}$ ) and subcutaneously (once daily). The surgical staples and sutures were removed $14-16 \mathrm{~d}$ and $26-28 \mathrm{~d}$ following surgery respectively.

\subsubsection{Termination}

At 52 weeks, the sheep were weighed and anesthetized. For each sheep, 3-4 $\mathrm{ml}$ of cerebrospinal fluid (CSF) was collected and kept at room temperature. The CSF samples were analyzed for the total amount of protein and fine cytology (red and white blood cell count). Moreover, for each sheep, approximately $2 \mathrm{ml}$ of blood was collected, kept at room temperature and analyzed for complete blood cell count and chemistry. The results were verified according to reference values [34]. The sheep were then euthanized by an IV injection of a pentobarbital solution (Dolethal ${ }^{\circledR}$, VETOQUINOL). The implants were extracted from the cranium by means of craniotome. Biopsies of different organs-lymph nodes, kidneys and liverwere taken to analyze if any implant-related toxicity was present.

\subsubsection{Histologic preparation}

The retrieved implants and an unimplanted control from each group were fixated in $10 \%$ neutral-buffered 
formalin, dehydrated in ethanol solution of increasing concentration, cleared in xylene, and finally embedded in polymethyl methacrylate (PMMA). After complete curing, the embedded implant was first cut in half transversally (blue section in figure 2) and the $\mathrm{CaP}-\mathrm{Ti}$ was also cut longitudinally (green section in figure 5). Sections were cut to an approximate thickness of $40 \mathrm{~mm}$ and a grinding technique was applied to reduce PMMA artifacts in the sections (EXAKT, Hamburg, Germany). The sections were stained with a polychromatic staining (modified Paragon).

\subsubsection{Histologic observations, quantification and} semi-quantitative histopathologic analysis

Sections and regions of interest (ROIs) analyzed in the histologic quantification are outlined in figure 2.

Four ROIs were analyzed: ROI 1 (lateral side), ROI 2 (medial side), ROI 3 (skin side) and ROI 4 (dura side). The ROIs for the quantification of each sample are available in the supplementary material (stacks.iop.org/BMM/16/035031/mmedia).

Each area was quantified for (a) bone-implant contact (BIC) (\%) corresponding to the osteointegration of the implant, defined as the percentage of the implant perimeter in direct contact with the mineralized bone tissue; (b) bone area (\%), defined as the percentage of the ROI occupied by bone tissue in terms of surface area, corresponding to the osteoconduction measured within the ROIs; (c) fibrous tissue area (\%) defined as the percentage of the ROI occupied by fibrous tissue; and (d) lacunae/adipose area (\%), defined as the percentage of the ROI occupied by either lacunae or adipose tissue.

Qualitative and semi-quantitative histopathologic evaluation of the local tissue effects and the performance was conducted for each implanted site. The analysis was conducted according to the ISO 10993-6 Standard [35]. The parameters were graded from 0 to 5 ( $0=$ absent, $1=$ minimal, $2=$ slight, $3=$ moderate $4=$ marked or $5=$ severe). Observations were made in conformance with the definition of newly formed bone (NB), per ASTM F2721 [36]. The inflammatory parameters were judged according standard above, with the addition of tissue response parameters as outlined by Zubery et al [37], Alves et al [38] and von Doernberg et al [39] to allow for a better characterization of the local tissue effects of the given test articles.

Horizontal sections of the CaP-Ti implant were performed in order to study the bone ingrowth and observe the ceramic material degradation (figure 5).

\subsection{Statistics}

The semi-quantitative histomorphometric data was evaluated for normality with the Shapiro-Wilk test, which shows non-normal distribution and therefore non-parametric statistics is applied.
Comparisons between CaP-Ti and Ti-mesh implant was done with paired, two-sided Wilcoxon signed-rank test. The histomorphometric parameters (BIC, bone area and fibrous and/or lacunae area) were calculated in percentages. Qualitative and semiquantitative parameters are presented in boxplots with individual data points presented as dots. $P$ values of $<0.05$ were considered statistically significant.

\section{Results}

\subsection{Ceramic characterization}

SEM micrographs illustrates the morphology of the ceramic. In figure 3(a) the general microstructure of the material can be observed. The porous structure is depicted in figure 3(b) and at high magnification in figure 3(c).

The phase composition of the obtained ceramic was a mixture of monetite $(86 \%), \beta$-TCP $(8 \%)$ and $\beta$ CPP $(6 \%)$. The XRD pattern is shown in figure $3(\mathrm{~d})$, indicating the peaks of all the present phases. The total porosity was measured as $43 \% \pm 1 \%$ and the surface area as $4 \mathrm{~m}^{2} \mathrm{~g}^{-1}$.

\subsection{Clinical pathology analysis}

\subsubsection{Surgery and termination}

The circular defects created during the surgery had a diameter of $14 \mathrm{~mm}$ on the top part and $11 \mathrm{~mm}$ on the bottom part (figure 4(c)), whilst the central depth of the defects ranged between $5 \mathrm{~mm}$ to $7 \mathrm{~mm}$ (median of 6). Both the Ti-mesh (figure 4(b)) and the CaP-Ti (figure 4(a)) implants covered the burrholes without complications (figure 4(d)). At termination, no skin abnormalities over the implantation sites were observed. No erythema, edema, swelling or signs of infection were observed in any sites. All the implantation sites demonstrated a thin coronal envelope that was either transparent or whitish with occasional red areas. A slight to moderate vascularization was noted for all implantation sites. Macroscopically all test articles were visible, integrated and no brain compressions were observed in any sheep. Meningocortical adhesions were noted in 2 out of 14 frontal sites with similar occurrence between $\mathrm{CaP}-\mathrm{Ti}$ and $\mathrm{Ti}$ mesh group ( 1 out of 7 sites for CaP-Ti and Ti-mesh groups).

\subsubsection{Titanium burr-hole cover}

Ti-meshes were associated with filling of the calvaria defect by fibrous tissue mainly. The soft tissue on top of the plug appeared dense and a thick layer was present in the created defect (figures 4(e) and (f)).

The inflammatory reaction associated with the Ti-mesh was discrete and no adverse effects were observed in the implanted site. Bone formation was recorded starting from the native bone (figure 4(e)), but no full closure was observed in any of the seven animals. No osteoconductivity or bone bridging was 


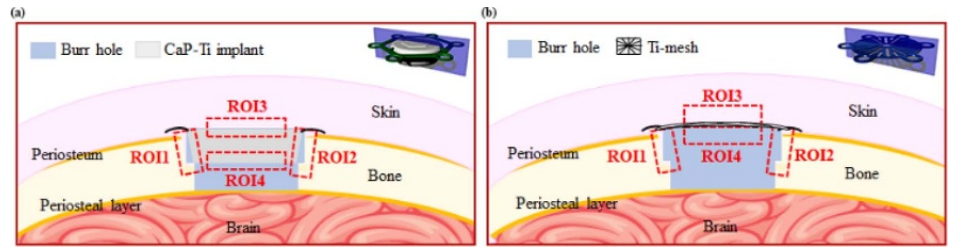

Figure 2. Scheme of the vertical histology cuts of (a) CaP-Ti device and (b) Ti-mesh burr-hole cover. Illustration of the regions of interest (ROIs) for (a) CaP-Ti and (b) for Ti mesh sections.
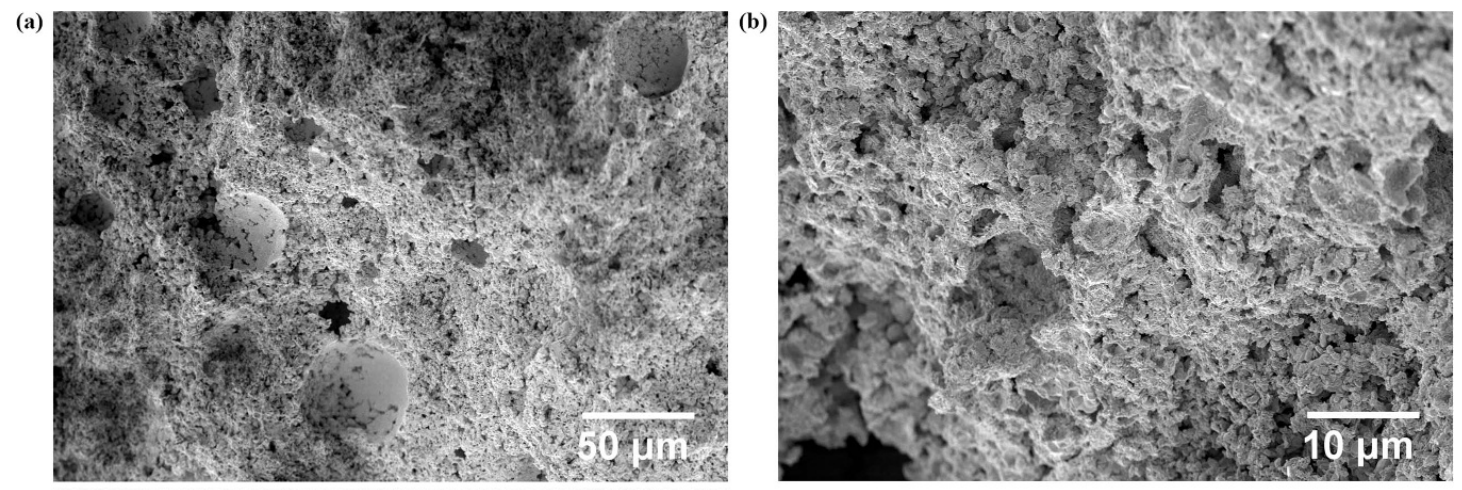

(c)

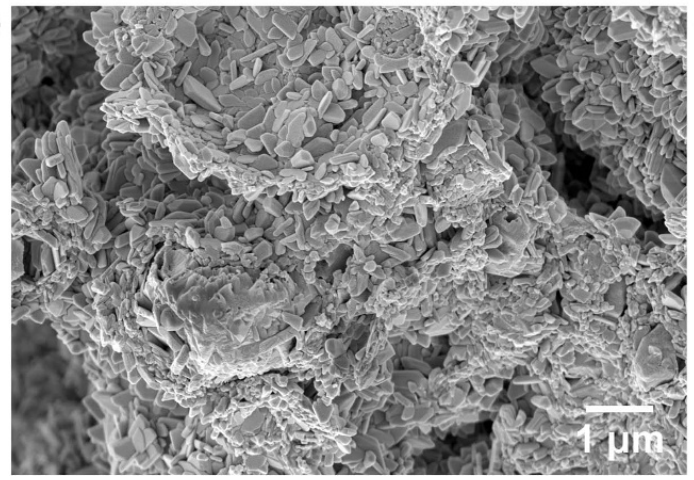

(d)

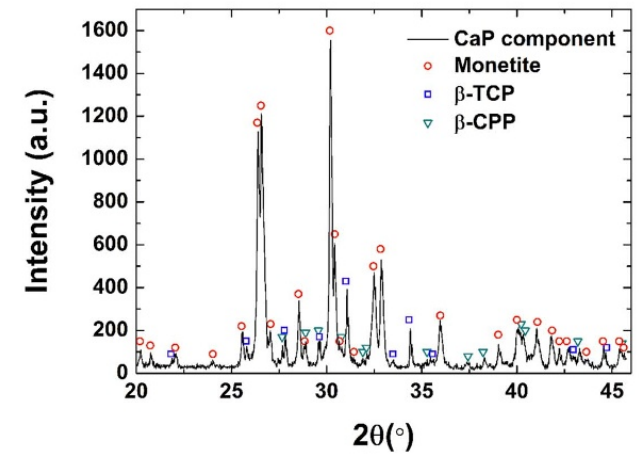

Figure 3. SEM micrographs at different magnifications show: (a) general microstructure of the ceramic including several pores, (b) morphology of the porous structure, (c) ceramic microstructure at high magnification. The XRD pattern of the ceramic component including the identified peaks labelled according to the corresponding phase present in the material (d).

observed in the proximity of the Ti-mesh which was encapsulated in fibrous tissue (figure $4(\mathrm{f})$ ). Bone formation occurred on the dura side and not in close vicinity to the mesh (figure $4(\mathrm{e})$ ).

\subsubsection{CaP-Ti implant}

After 52 weeks of implantation, the CaP-Ti implant was characterized by a moderate degradation of the $\mathrm{CaP}$ part (figure $4(\mathrm{a})$ vs figure $4(\mathrm{~g})$ ) that was replaced by mature bone tissue with a structure similar to the hosts' bone (figure $4(\mathrm{~g})$ ). The inflammatory reaction associated with the CaP-Ti implant was discrete. $\mathrm{CaP}$ degradation was attested histologically by the presence of material-filled macrophages (MFM) and affected all article sides (figure 4(h)); however, the degradation looked more pronounced on the skin side, where only a thin layer of bone was observed. On the other hand, bone formation was more evident on the dura side, where osseous bridging from the sides was unilateral in 1 of 7 sites and bilateral in 6 of 7 sites in the CaP-Ti implant (see example in figure $4(\mathrm{~g})$ ); and NB was present inside the pores of the material and at locations where the material had degraded (figure 4(h)).

Fibrous tissue was present especially on the top of the implant and where parts of the Ti skeleton were exposed (figure $4(\mathrm{~g})$ ).

In the horizontal sections (figure 5), it is possible to observe how the degraded material was substituted with the bone ingrowth into the $\mathrm{CaP}$ pores (figures 5(b) and (c)). It is also possible to distinguish MFM trapped in the adipose tissue of the NB (figures 5(c) and (d)). In the high magnification horizontal and vertical sections (figures 5(e) and (f)) the material-filled cells can be clearly identified as macrophages.

\subsubsection{Histologic quantification}

Significantly higher bone-implant contact (figure 6(a)) was found for the CaP-Ti implant in 


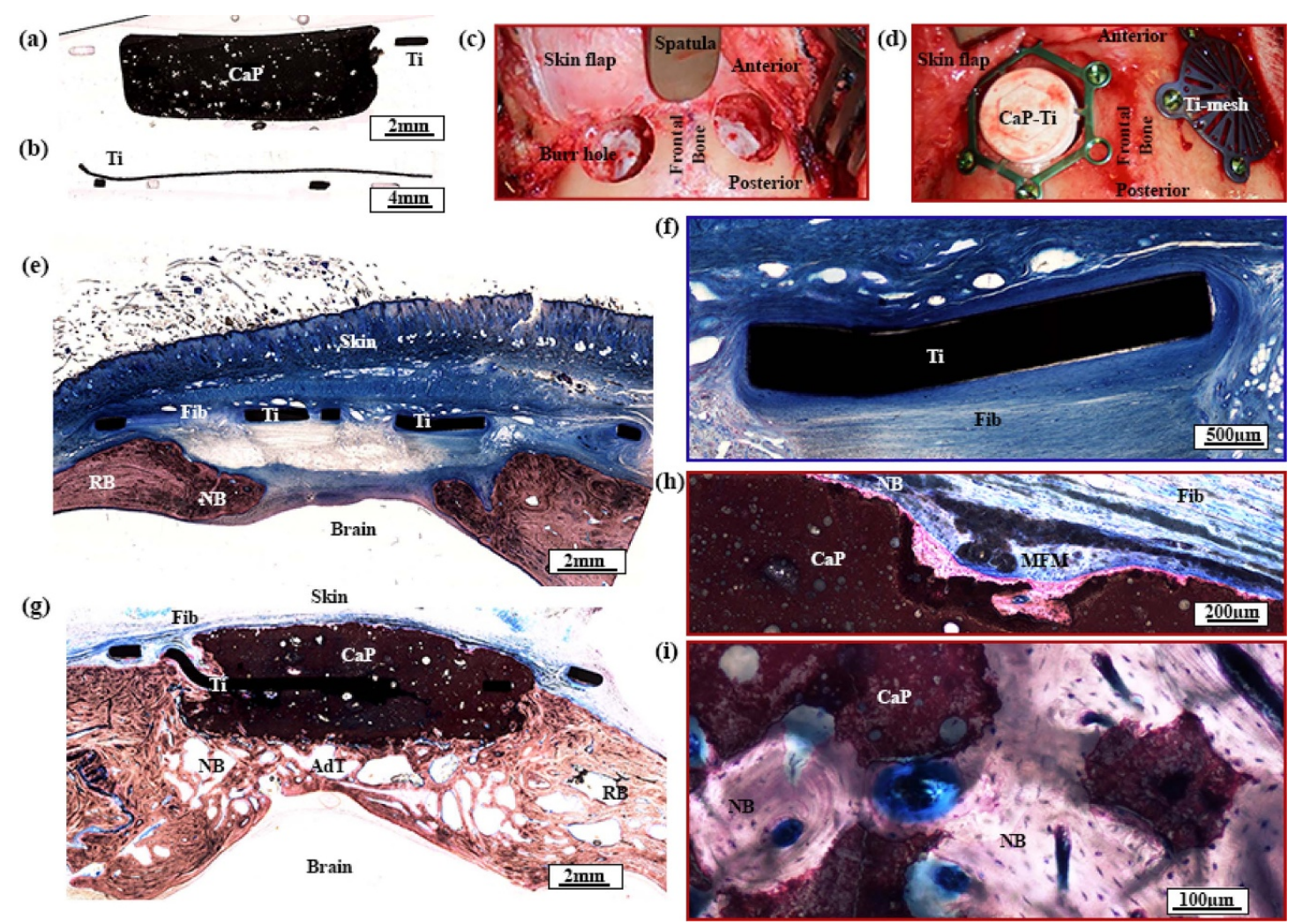

Figure 4. Vertical section: unimplanted CaP-Ti (a) and Ti-mesh (b); and perioperative images of burr-holes (c) and implanted CaP-Ti and Ti-mesh burr-hole covers (d). Modified-paragon stained histologies of the implanted Ti-mesh at low (e) and high magnification (f) (zoom of (e)) and the corresponding CaP-Ti at low (g) and high magnification (h) and (i) (zoom of (g)). Acronyms: CaP: calcium phosphate, AdT: adipose tissue, Fib: fibrous tissue, MFM: material-filled macrophages, RB: recipient bone, NB: newly formed bone, Ti: titanium.

comparison to the Ti-mesh in all ROIs $(\mathrm{p}<0.001$ on the dura side, $\mathrm{p}<0.05$ on the bone edge and skin side). Very high bone-implant contact was observed specifically in the defect edge regions (ROI 1 and 2) and on the dura side (ROI 4) for the CaP-Ti implant compared to the Ti-mesh where no bone had formed in direct contact with the Ti-mesh with one exception in ROI 1. Overall, BIC quantification showed good osteointegration of the whole CaPTi implant with seemingly close direct, adherent and strong bonding with the bone in direct contrast to the Ti-mesh burr-hole cover. A lot of NB was found in the implant-defect border region (ROI 1 and 2) for both implants, but significantly more bone had formed for the CaP compared to the Timesh-especially on the dura but also on the skin side $(\mathrm{p}<0.05)$.

Significant difference in the fibrous tissue formation (figure 6(c)) was observed for the Ti-mesh as compared to the CaP-Ti implant in all ROIs $(p<0.05)$. The Ti-mesh burr-hole defects were filled with fibrous tissue mainly whilst for the CaP-Ti, fibrous infiltration was limited and restricted to the defect borders. A significant higher lacunae/adipose area (figure 6) was found for the CaP-Ti implant only on the dura side $(p<0.05)$. The lacunae/adipose area was counted separate from the bone area even though the host bone tissue presents with a similar structure and it was found mostly within the NB (figure $4(\mathrm{~g})$ ).

\subsubsection{Histopathologic analysis}

The results of the semi-quantitative histopathologic analysis are presented in figure 7 . Significantly higher bone neoformation $(\mathrm{p}<0.001)$ with moderate osteoconduction $(\mathrm{p}<0.001)$ and a marked osteointegration $(\mathrm{p}<0.001)$ were observed in the CaP-Ti cover versus the Ti-mesh. A non-significant difference in the presence of osteoblastic cells $(p>0.05)$ was found. Noteworthy is the significantly larger presence of macrophages $(\mathrm{p}<0.001)$ and lower fibrosis/encapsulation $(\mathrm{p}<0.001)$ in CaP-Ti implant compared to the Ti-mesh. A minimal to absent amount of multinucleated giant cells were observed in both materials with a slightly larger amount observed for the $\mathrm{CaP}-\mathrm{Ti}$ implant $(\mathrm{p}<0.05)$. Partial degradation of the calcium phosphate with replacement of mature bone tissue was found in the Ca-Ti implant and no tissue necrosis was detected for any implant in the study.

\subsubsection{Systemic and local effects}

Blood and CSF analysis showed normal levels in all animals. 

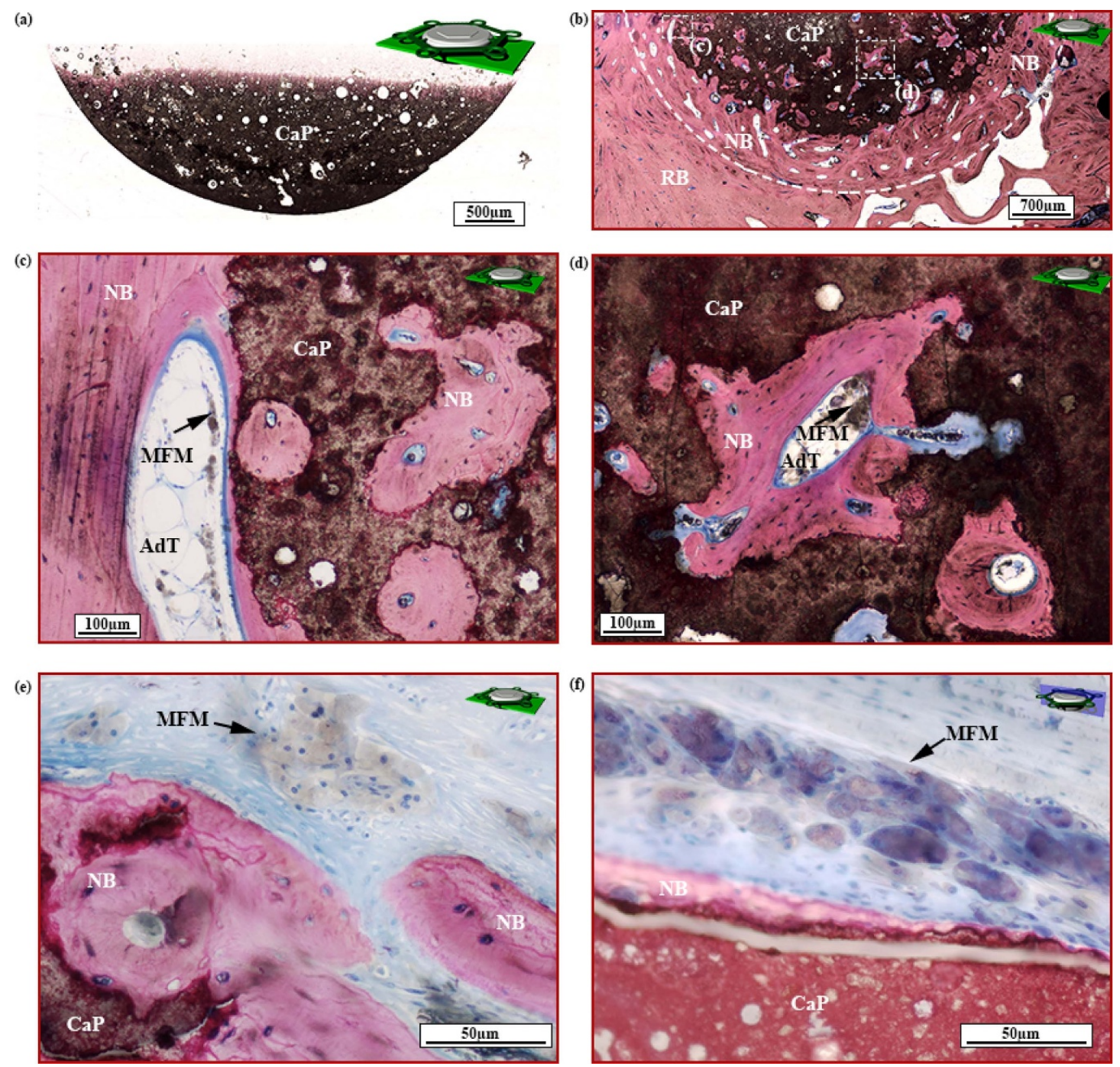

Figure 5. Cross-section of the CaP-Ti implant before (a) and after 52 weeks (b) implantation in a sheep model (white dashed line representing the original defect). In (c) and (d) zoom of area of (b). High magnification of MFM in horizontal (e) and vertical section (f). Acronyms: CaP: calcium phosphate, AdT: adipose tissue, MFM: material-filled macrophages, RB: recipient bone, NB: newly formed bone.

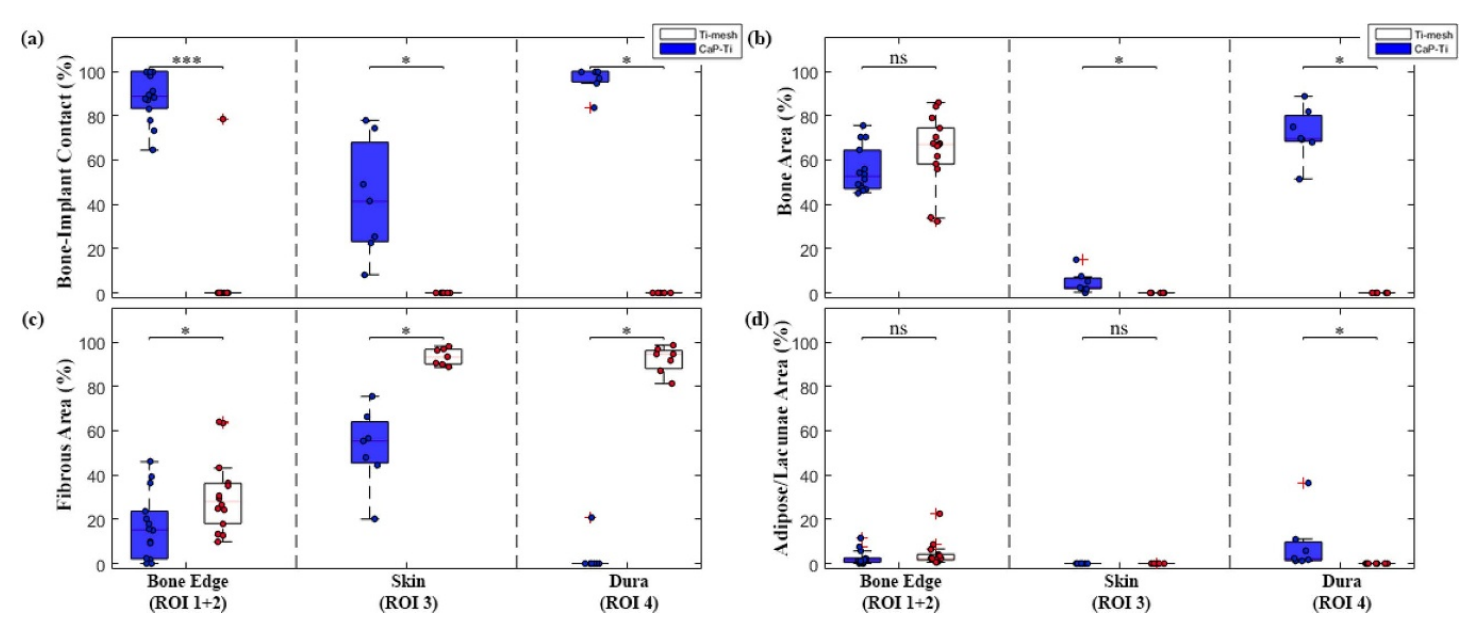

Figure 6. Semi-quantification of the different ROIs where all data are presented with dots (blue for CaP-Ti and red for Ti-mesh) and their median and distribution are represented in boxplots (blue for CaP-Ti and white for Ti-mesh). The parameters analyzed were: (a) bone-implant contact (\%), (b) bone area (\%), (c) fibrous area (\%) and (d) adipose/lacunae area (\%). 


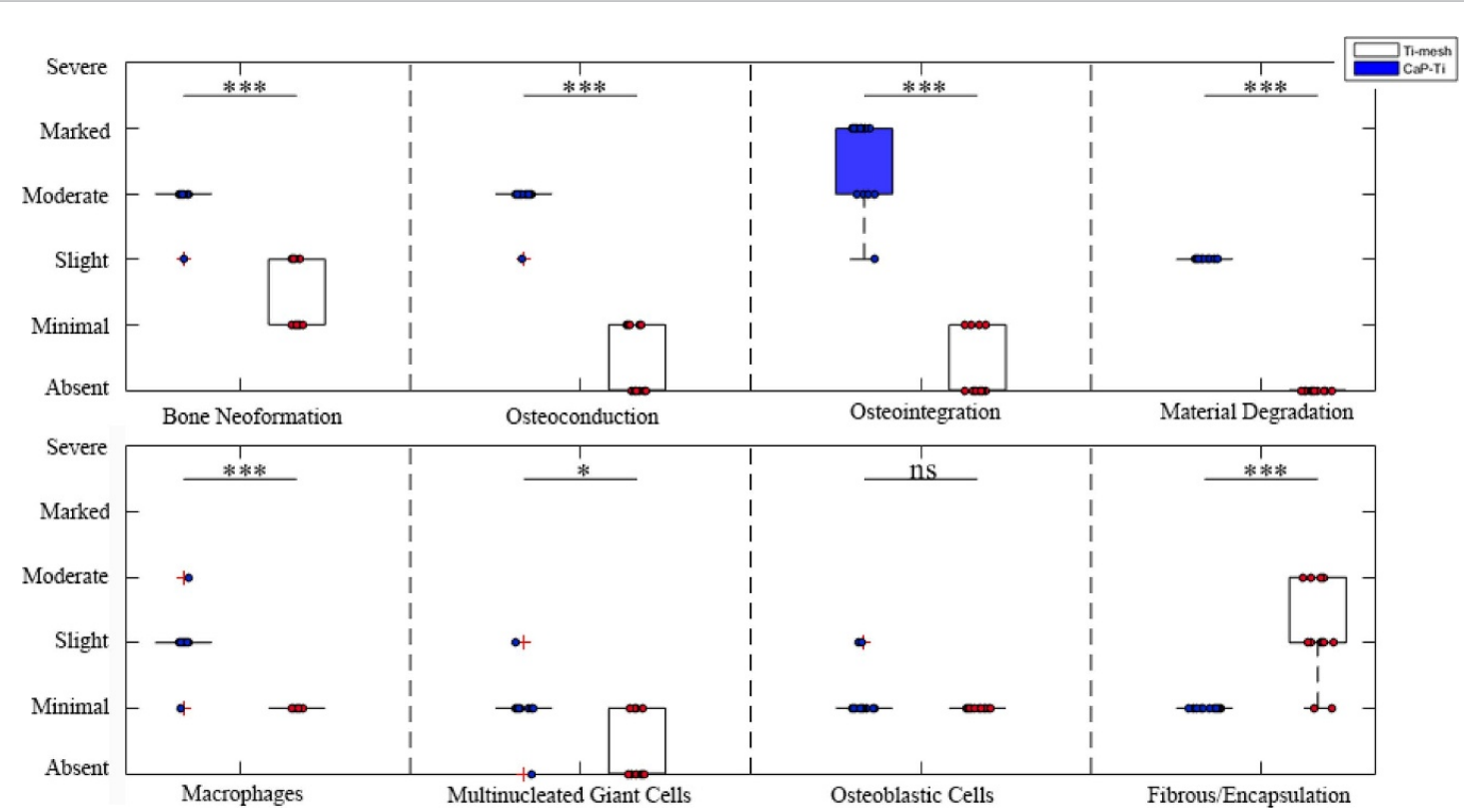

Figure 7. Summary of the semi-quantitative histopathologic analysis after 52 weeks $(n=7)$ on vertical sections. The evaluation was based on the general rules of pathology, with findings being graded 0 (absent), 1 (minimal), 2 (slight), 3 (moderate), 4 (marked) or 5 (severe). The data were represented as dots (blue for CaP-Ti and red for Ti-mesh) and boxplots (blue for CaP-Ti and white for Ti-mesh). The parameters evaluated were: bone neoformation, osteoconduction, osteointegration, material degradation, macrophages, multinucleated giant cells, osteoblastic cells, and fibrosis/encapsulation. $\left({ }^{* * *}: \mathrm{p}<0.001,{ }^{*}: \mathrm{p}<0.05\right.$, ns: $\mathrm{p}>0.05)$.

Presence of meningo-cortical adhesion was noted in 1 out 7 implants for both CaP-Ti and Ti-mesh. However, no treatment-related histopathologic findings were shown in the brain. No dura lesion was noted for either the CaP-Ti implant or the Ti-mesh. No implant-related toxicity was found in either draining lymph nodes, liver or kidney biopsies. The inflammatory reaction associated with the both implants were discrete, and no adverse effects were observed in the implanted sites.

\section{Discussion}

In this study, we found that the CaP-Ti reinforced burr-hole cover significantly improved bone formation and tissue response after 52 weeks of implantation, whilst the Ti-mesh exhibited a thick fibrous tissue with minimal to absent osteointegration or osteoconduction. None of the open cranial defects underneath the Ti-mesh had fused in comparison to the CaP-Ti implant, where bilateral fusion was observed in 6 out of 7 defects and unilateral in the last site. Even though no bone was found in the proximity of the Ti-mesh, bone formation was evident at the bone edges in close vicinity to the dura. This behavior is in line with the CaP-Ti device where significantly more bone had formed on the dura side as compared to the skin side, which could be explained by two facts: first, the vitality of the periosteum and periosteal layer of the dura mater plays a key role in bone regeneration; and second, the periosteum is damaged during surgery whilst the periosteal layer remains intact [4044]. In essence, the preservation of the periosteum and periosteal layer of the dura seems to be vital for the restoration of cranial defects and the lack thereof will impact bone regeneration long term.

The high bone-implant contact found in CaPTi implants (64\%-100\% bone edge and 84\%-100\% dura) indicates that the $\mathrm{NB}$ has a direct adherent and strong bond to the CaP. This close bond is not only observed on the surface of the material but also inside the pores of the material. The monetite, $\beta$ TCP, and calcium pyrophosphate $\mathrm{CaP}$ component degraded slightly and was to a large extent replaced by mature viable bone with a structure similar to that of the host animal, exhibiting good osteointegration and osteoconduction (figure 4).

Osteointegration is a complex multifactorial immune-modulated healing process with the involvement of numerous different cells and mediators [37]. The long-term clinical outcome is dependent on obtaining homeostasis that, if disturbed, can induce additional activation of the immune system, causing an interruption of the osteointegration of the material with the host bone. The presence of material filled macrophages after 1 year indicates that macrophages play a key role in active cell-mediated resorption and bone regeneration in $\mathrm{CaP}$ cements, not only as previously described in the initial stages (weeks) [15, 45-47] and mid-term (up to 6 months) [15] but potentially also long term (years). Even though the triggering factors of the body-reaction to the implant and macrophages recruitment is not fully known, $\beta$-TCP and pyrophosphate may be released by the faster resorption of the crystalline monetite matrix [18] and seem to be good candidates for macrophage 
triggering factors $[48,49]$. In particular, pyrophosphate seems to be a potent mitogen, a critical regulator in bone mineralization, and it has displayed the capability of stimulating proliferation in multiple cell types [48].

After 52 weeks, only few osteoblasts and multinucleated giant cells were observed in all implants. For the CaP-Ti implant, this is likely coupled with the significant amount of new viable bone that has formed in the defect and that natural remodeling and homeostasis have set in; whilst for the Ti-mesh, this simply reflects the slow growth of the natural bone in open cranial defects. Bone did not only form on the surface of the CaP-Ti implant but was also observed in its micro- and macro-pores. Material resorption either due to passive dissolution or to cellmediated resorption leads to the release of calcium ions into the protected microenvironment, triggering bone formation [50]. Previous studies with $\mathrm{CaP}$ cements have observed mineralized tissue or bone within pores [51-53]; however, no previous observations have been made of MFM inside the pores. It is likely that the macrophages migrated into the material through interconnected pores as part of an immune-orchestrated osteogenesis, which is indicated by the bone found adjacent to the MFM.

Clinically, Vasella et al [54] showed that it is more favorable to place a burr-hole cover than leaving it open. The perceived aesthetical result improved after the placement of a burr-hole cover without additional pain or infections $[54,55]$. Without a burr-hole cover, the soft tissue will sink into the defect, causing, on top of the aesthetical problem, impairment of bone formation and consequently incomplete bony closure. For the Ti-mesh in this study, the burr-hole defects were mainly filled with fibrous tissue and minimal to absent osteointegration or osteoconduction was observed. By embedding the Ti-mesh in a CaP ceramic, the amount of fibrous tissue formed was significantly lowered. Furthermore, stable anchoring of the implant by direct bone-implant contact to the recipient's bone as well as bony fusion of the defect was achieved.

A limitation of the present study is that no open defect was used as a control. Therefore, only an assumption can be made that the bony growth seen in the burr-hole defects is unrelated to the Ti-mesh cover. However, similar bony growth on the dura side was corroborated in long-term CT follow-up in patients having undergone craniofacial reconstruction without the use of a burr-hole cover (L Kihlström Burenstam Linder, MD, unpublished data, 2020).

CaP-Ti implants revealed osteointegration to a larger extent than Ti-mesh, where the viable bone was clearly visible in the defects with bone tissue migrating into existing pores, replacing resorbed $\mathrm{CaP}$ material. These results warrant in-depth studies addressing the mechanism behind the good osteointegration. Future work will focus on the underlying biological mechanisms in terms of how and when specific cell types are recruited.

\section{Conclusions}

The integration of the $\mathrm{Ti}$-mesh in a $\mathrm{CaP}$ improved bone formation and osteointegration in comparison to a bare Ti-mesh. The bare Ti-mesh burr-hole covers were associated with filling of the calvarial defect by fibrous tissue mainly whilst CaP-Ti was replaced with mature bone during the healing process. Significantly more bone formed on the dura side highlighting its importance in osteogenesis.

The long-term presence of viable macrophages indicates that they play an important role in material degradation, bone formation and homeostasis and should be studied further.

\section{Acknowledgments}

We are grateful for the funding provided by the BIOMATCELL Vinn Excellence Center of Biomaterials and Cell Therapy, Gothenburg, Sweden. Associate Professor Omar Omar, University of Gothenburg, Professor Peter Thomsen, University of Gothenburg and Associate Professor Thomas Engstrand, Uppsala University, are acknowledged for their valuable contributions to background theory, design of experiment, device protype and data acquisition. MSc Elodie Royet and her team at NAMSA (France) for the conduction of the animal study.

The work was supported by Vinnova (Grant No. 2015-04533); the Swedish Research Council (Grant No. 2017-04728); and Swedish Foundation for Strategic Research (Grant No. RMA15-0110).

Drs Gallinetti and Birgersson report personal fees from OssDsign both during the conduct of the study and outside the submitted work. Drs Kihlström, Åberg and Engqvist have consulting agreements with OssDsign. Drs Engqvist and Åberg have direct ownership in OssDsign. Dr Illies has no financial disclosures.

\section{ORCID iDs}

Sara Gallinetti (i) https://orcid.org/0000-0001-76489250

Lars Kihlström Burenstam Linder (1)

https://orcid.org/0000-0002-7918-9787

Håkan Engqvist (1) https://orcid.org/0000-00019529-650X

Ulrik Birgersson (i) https://orcid.org/0000-0002-

7983-925X

\section{References}

[1] Neovius E and Engstrand T 2010 Craniofacial reconstruction with bone and biomaterials: review over the last 11 years $J$. Plast. Reconstr. Aesthet. Surg. 63 1615-23 
[2] Moreira-Gonzalez A, Jackson I T, Miyawaki T, Barakat K and DiNick V 2003 Clinical outcome in cranioplasty: critical review in long-term follow-up J. Craniofac. Surg. 14 144-53

[3] van de Vijfeijken S E C M et al 2018 Autologous bone is inferior to alloplastic cranioplasties: safety of autograft and allograft materials for cranioplasties, a systematic review World Neurosurg. 117 443-52

[4] Honeybul S, Morrison D A, Ho K M, Lind C R P and Geelhoed E 2016 A randomized controlled trial comparing autologous cranioplasty with custom-made titanium cranioplasty J. Neurosurg. $1261-10$

[5] Kwarcinski J, Boughton P, Ruys A, Doolan A and van Gelder J 2017 Cranioplasty and craniofacial reconstruction: a review of implant material, manufacturing method and infection risk Appl. Sci. 7 1-17

[6] Yang J, Sun T, Yuan Y, Li X, Yu H and Guan J 2019 Evaluation of titanium mesh cranioplasty and polyetheretherketone cranioplasty: protocol for a multicentre, assessor-blinded, randomised controlled trial BMJ Open 9 1-6

[7] Thien A, King N K K, Ang B T, Wang E and Ng I 2015 Comparison of polyetheretherketone and titanium cranioplasty after decompressive craniectomy World Neurosurg. 83 176-80

[8] Zhang Q, Yuan Y, Li X, Sun T, Zhou Y, Yu H and Guan J 2018 A large multicenter retrospective research on embedded cranioplasty and covered cranioplasty World Neurosurg. 112 e645-51

[9] Roh H, Kim J, Kim J H, Chong K, Yoon W K, Kwon T-H and Kim J H 2019 Analysis of complications after cranioplasty with a customized three-dimensional titanium mesh plate World Neurosurg. 123 e39-44

[10] Yoshioka N and Tominaga S 2018 Titanium mesh implant exposure due to pressure gradient fluctuation World Neurosurg. 119 e734-9

[11] Mendes L S, Saska S, Coelho F, Capote T S D O, Scarel-Caminaga R M, Marchetto R, Carrodeguas R G, Gaspar A M M and Rodríguez M A 2018 Injectable $\beta$-TCP/MCPM cement associated with mesoporous silica for bone regeneration: characterization and toxicity evaluation Biomed. Mater. 13025023

[12] LeGeros R Z 2002 Properties of osteoconductive biomaterials: calcium phosphates Clin. Orthop. Relat. Res. 395 81-98

[13] Dorozhkin S V 2007 Bioceramics based on calcium orthophosphates (review) Glas. Ceram. 64 442-7

[14] Lu J, Descamps M, Dejou J, Koubi G, Hardouin P, Lemaitre J and Proust J-P 2002 The biodegradation mechanism of calcium phosphate biomaterials in bone J. Biomed. Mater. Res. 63 408-12

[15] Kuemmerle J M, Oberle A, Oechslin C, Bohner M, Frei C, Boecken I and Rechenberg B V 2005 Assessment of the suitability of a new brushite calcium phosphate cement for cranioplasty-an experimental study in sheep $J$. Cranio-Maxillofacial Surg. 33 37-44

[16] Dorozhkin S V 2011 Calcium orthophosphates: occurrence, properties, biomineralization, pathological calcification and biomimetic applications Biomatter 1 121-64

[17] Tamimi F, Sheikh Z and Barralet J 2012 Dicalcium phosphate cements: brushite and monetite Acta Biomater. 8 474-87

[18] Bohner M, Theiss F, Apelt D, Hirsiger W, Houriet R, Rizzoli G, Gnos E, Frei C, Auer J A and von Rechenberg B 2003 Compositional changes of a dicalcium phosphate dihydrate cement after implantation in sheep Biomaterials 24 3463-74

[19] Gbureck U, Hölzel T, Klammert U, Würzler K, Müller F and Barralet J 2007 Resorbable dicalcium phosphate bone substitutes prepared by $3 \mathrm{D}$ powder printing Adv. Funct. Mater. 17 3940-5

[20] Sheikh Z, Zhang Y L, Grover L, Merle G E, Tamimi F and Barralet J 2015 In vitro degradation and in vivo resorption of dicalcium phosphate cement based grafts Acta Biomater. $26338-46$
[21] Bohner M, Galea L and Doebelin N 2012 Calcium phosphate bone graft substitutes: failures and hopes J. Eur. Ceram. Soc. 32 2663-71

[22] Kihlström Burenstam Linder L, Birgersson U, Lundgren K, Illies C and Engstrand T 2019 Patient-specific titanium-reinforced calcium phosphate implant for the repair and healing of complex cranial defects World Neurosurg. 122 e399-407

[23] Persson J, Helgason B, Engqvist H, Ferguson S J and Persson C 2018 Stiffness and strength of cranioplastic implant systems in comparison to cranial bone $J$. Cranio-Maxillofac. Surg 46 418-23

[24] Engstrand T, Kihlström L, Lundgren K, Trobos M, Engqvist $\mathrm{H}$ and Thomsen P 2015 Bioceramic implant induces bone healing of cranial defects Plast. Reconstr. Surg. Glob. Open 3 1-4

[25] Engstrand T, Kihlström L, Neovius E, Skogh A-C D, Lundgren T K, Jacobsson H, Bohlin J, Åberg J and Engqvist H 2014 Development of a bioactive implant for repair and potential healing of cranial defects J. Neurosurg. $120273-7$

[26] Doebelin N and Kleeberg R 2015 Computer programs Profex: a graphical user interface for the Rietveld refinement program BGMN J. Appl. Crystallogr. 48 1573-80

[27] Dickens B, Schroeder L W and Brown W E 1974 Crystallographic studies of the role of $\mathrm{Mg}$ as a stabilizing impurity in $\beta$-Ca3(PO4)2. The crystal structure of pure 及-Ca3(PO4)2 J. Solid State Chem. 10 232-48

[28] Schroeder L W, Prince E and Dickens B 1975 Hydrogen bonding in $\mathrm{Ca}(\mathrm{H} 2 \mathrm{PO} 4) 2 \cdot \mathrm{H} 2 \mathrm{O}$ as determined by neutron diffraction Acta Crystallogr. B 31 9-12

[29] Curry N A and Jones D W 1971 Crystal structure of brushite, calcium hydrogen orthophosphate dihydrate: a neutron-diffraction investigation J. Chem. Soc. A 3725

[30] Boudin S, Grandin A, Borel M M, Leclaire A and Raveau B 1993 Redetermination of the $\beta$-Ca2P2O7 structure Acta Crystallogr. C 49 2062-4

[31] Dickens B, Bowen J S and Brown W E 1972 A refinement of the crystal structure of CaHPO4 (synthetic monetite) Acta Crystallogr. B 28 797-806

[32] Brunauer S, Emmett P H and Teller E 1938 Adsorption of gases in multimolecular layers J. Am. Chem. Soc. 60 309-19

[33] Ajaxon I, Maazouz Y, Ginebra M P, Öhman C and Persson C 2015 Evaluation of a porosity measurement method for wet calcium phosphate cements J. Biomater. Appl. 30 526-36

[34] Wilhelmi M H, Tiede A, Teebken O E, Bisdas T, Haverich A and Mischke R 2012 Ovine blood Asaio J. $5879-82$

[35] ISO 10993-6:2016 2016 Biological Evaluation of Medical Devices-Part 6: Tests for Local Effects after Implantation (Geneva: ISO copyright office)

[36] ASTM F2721-09(2014) 2014 Standard Guide for Pre-clinical in Vivo Evaluation in Critical Size Segmental Bone Defects (West Conshohocken: ASTM International)

[37] Zubery Y, Goldlust A, Alves A and Nir E 2007 Ossification of a novel cross-linked porcine collagen barrier in guided bone regeneration in dogs J. Periodontol. 78 112-21

[38] Alves A and Boutrand J-P 2003 Biocompatibilty and performance of an interbody resorbable fusion cage implanted in functional site in sheep Eur. Cells Mater. 546

[39] von Doernberg M C, von Rechenberg B, Bohner M, Grünenfelder S, van Lenthe G H, Müller R, Gasser B, Mathys R, Baroud G and Auer J 2006 In vivo behavior of calcium phosphate scaffolds with four different pore sizes Biomaterials 27 5186-98

[40] Reid C A, McCarthy J G and Kolber A B 1981 A study of regeneration in parietal bone defects in rabbits Plast. Reconstr. Surg. 67 591-6 
[41] Duchamp De Lageneste O, Julien A, Abou-Khalil R, Frangi G, Carvalho C, Cagnard N, Cordier C, Conway S J and Colnot C 2018 Periosteum contains skeletal stem cells with high bone regenerative potential controlled by Periostin Nat. Commun. 9 1-15

[42] Roberts S J, van Gastel N, Carmeliet G and Luyten F P 2015 Uncovering the periosteum for skeletal regeneration: the stem cell that lies beneath Bone 70 10-18

[43] Hobar C P, Masson J A, Wilson R and Zerwekh J 1996 The importance of the dura in craniofacial surgery Plast. Reconstr. Surg. 98 217-25

[44] Spector J A, Greenwald J A, Warren S M, Bouletreau P J, Crisera F E, Mehrara B J and Longaker M T 2002 Co-culture of osteoblasts with immature dural cells causes an increased rate and degree of osteoblast differentiation Plast. Reconstr. Surg. 109 631-42

[45] Constantz B R et al 1998 Histological, chemical, and crystallographic analysis of four calcium phosphate cements in different rabbit osseous sites J. Biomed. Mater. Res. 43 451-61

[46] Schlundt C et al 2018 Macrophages in bone fracture healing: their essential role in endochondral ossification Bone 106 78-89

[47] Theiss F, Apelt D, Brand B, Kutter A, Zlinszky K, Bohner M, Matter S, Frei C, Auer J A and von Rechenberg B 2005 Biocompatibility and resorption of a brushite calcium phosphate cement Biomaterials 26 4383-94

[48] Pujari-Palmer M, Pujari-Palmer S, Lu X, Lind T, Melhus H, Engstrand T, Karlsson-Ott M and Engqvist H 2016 Pyrophosphate stimulates differentiation, matrix gene expression and alkaline phosphatase activity in osteoblasts PloS One 11 e0163530

[49] Arbez B, Manero F, Mabilleau G, Libouban H and Chappard D 2019 Human macrophages and osteoclasts resorb $\beta$-tricalcium phosphate in vitro but not mouse macrophages Micron 125102730

[50] Ripamonti U, Roden L C, Ferretti C and Klar R M 2011 Biomimetic matrices self-initiating the induction of bone formation J. Craniofac. Surg. 22 1859-70

[51] Bohner $\mathrm{M}$ et al 2017 Characterization and distribution of mechanically competent mineralized tissue in micropores of $\beta$-tricalcium phosphate bone substitutes Mater. Today 20 106-15

[52] Draenert M, Draenert A and Draenert K 2013 Osseointegration of hydroxyapatite and remodeling-resorption of tricalciumphosphate ceramics Microsc. Res. Tech. 76 370-80

[53] Sweedy A, Bohner M and Baroud G 2018 Multimodal analysis of in vivo resorbable $\mathrm{CaP}$ bone substitutes by combining histology, SEM, and microcomputed tomography data J. Biomed. Mater. Res. B 106 1567-77

[54] Vasella F, Akeret K, Smoll N R, Germans M R, Jehli E, Bozinov O, Regli L and Stienen M N et al 2018 Improving the aesthetic outcome with burr hole cover placement in chronic subdural hematoma evacuation - a retrospective pilot study Acta Neurochir. 160 2129-35

[55] Im T, Lee Y and Suh S 2014 The efficacy of titanium burr hole cover for reconstruction of skull defect after burr hole trephination of chronic subdural hematoma Korean J. Neurotrauma 10 76-81 